\section{JTI}

JOURNAL OF

TRAUMA AND INJURY

\title{
The Successful Removal of a Foreign Body in the Spleen via Diaphragm Laceration Site by Video-Assisted Thoracoscopic Surgery
}

\author{
Yang Bin Jeon, M.D., Ph.D., Sung Youl Hyun, M.D., Ph.D., Dae Sung Ma, M.D. \\ Department of Trauma Surgery, Trauma Center, Gachon University Gil Medical Center, \\ Incheon, Korea
}

Received: December 17, 2018

Revised: June 23, 2019

Accepted: June 25, 2019

\section{Correspondence to}

Dae Sung Ma, M.D.

Department of Trauma Surgery, Gil Medical Center, Gachon University College of

Medicine, 21 Namdong-daero 774beon-

gil, Namdong-gu, Incheon 21565, Korea

Tel: +82-32-460-3010

Fax: $+82-32-460-2372$

E-mail:pegamu@gilhospital.com

A 73-year-old man, who, in an inebriated state, had slipped in a flowerbed and was wounded on the left flank, was transferred to Trauma Center, Gil Medical Center, Gachon University College of Medicine. Based on the chest and abdominopelvic computed tomography, he was diagnosed with multiple rib fractures and hemopneumothorax on the left hemithorax and was found to have a bony fragment in the spleen. He had not presented peritonitis and exsanguinous symptoms during the observation period. Seven days later, computed tomography of the abdomen showed suspected diaphragmatic injury and a retained foreign body in the spleen. On exploration by video assisted thoracoc surgery (VATS), a herniated omentum through the lacerated site of the diaphragm was observed. After omentectomy using Endo Gia, the foreign body in the spleen was observed through the lacerated site of the diaphragm. Traumatic diaphragm rupture with a foreign body, in the spleen, was successfully managed by video assisted thoracic surgery via the lacerated site of the diaphragm.

Keywords: Diaphragm; Foreign bodies; VATS

\section{INTRODUCTION}

The incidence of penetrating diaphragmatic injuries associated with the thoracoabdominal area is $11-19 \%$ [1]. A retained foreign body in a penetrating abdominal injury is a relative indication of laparotomy. However, delayed diagnosis of traumatic diaphragmatic rupture is associated with high morbidity and mortality in the long-term. Thoracoscopic exploration of suspected diaphragm injury without peritonitis sign 
and other peritoneal organs may be a reliable approach for exploration and management of traumatic diaphragm injury. In the thoracoscopic approach, it has a limitation for exploration of the pertitoneal cavity, but it is possible through the lacerated site in the diaphragm.

\section{CASE REPORT}

A 73-year-old man, who, in an inebriated state, had slipped down on a flowerbed and was wounded on the left flank, was transferred to Trauma Center, Gil Medical Center, Gachon University College of Medicine. In the previous hospital, computed tomography (CT) of the chest and abdominopelvic (AP) region were evaluated and revealed moderate hemopneumothorax on the left hemithorax with multiple rib fractures from 9th to 12th, fracture of the spinous process of T12 and L1, and spleen laceration due to the presence of a bony fragment (Fig. 1). The vital signs of the patient were stable without signs of peritonitis. He was admitted to the Trauma Intensive Care Unit after a closed thoracostomy on the left hemithorax and a primary closure on the flank wound (about $5 \mathrm{~cm}$ ). After 1 day, he was transferred to the general ward and was conservatively managed. The chest tube was removed after 5 days. He was stable without signs or symptoms of peritonitis during observation period. After 7 days, he had a follow-up AP CT which revealed improving subcapsular hematoma of the spleen and retention of foreign body. However, compared with the previous AP CT, it was observed that there was an increase in the fat density in the thoracic cavity above the spleen and remainder of the hemothorax (Fig. 2). A diaphragmatic injury was suspected. Video-assisted thoracic surgery (VATS) for exploration of the diaphragm was performed. After the evacuation of retained hematoma $(300 \mathrm{~mL})$, a herniated omentum was observed through the lacerated site of the diaphragm, which was approximately $4 \mathrm{~cm}$. After resection of omentum using an endoscopic stapler, a foreign body in the spleen was observed directly below the lacerated site of the diaphragm. After removal of the foreign body, no exsanguination or complications related to the procedure were observed in the spleen. The diaphragm was repaired by interrupted sutures using 2-0 PROLENE ${ }^{\circledR}$ Polypropylene Suture (Ethicon Inc; Johnson \& Johnson, Somerville, NJ, USA). The foreign body, which was suspected to be a bony fragment, was identified as a broken piece of the flowerpot (Fig. 3). He was discharged 14 days post-surgery without complications.

\section{DISCUSSION}

The incidence of penetrating diaphragmatic injuries associated with the thoracoabdominal area is $11-19 \%$ [1]. Penetrating injuries in the abdomen are generally treated by laparotomy, but in some cases, without hemodynamic instability and signs of peritonitis, conservative manage-
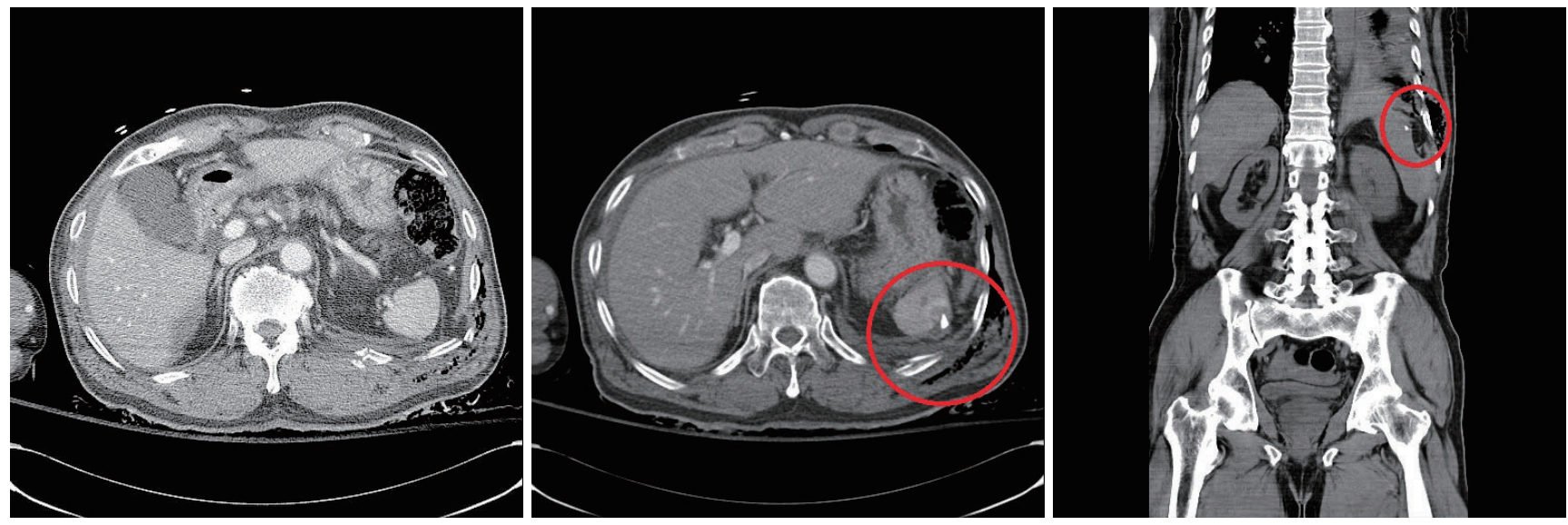

Fig. 1. Chest computed tomography (CT) and abdominopelvic CT finding, red circle indicates a foreign body like a bone fragment in the spleen and subcapsular hematoma in initially. 

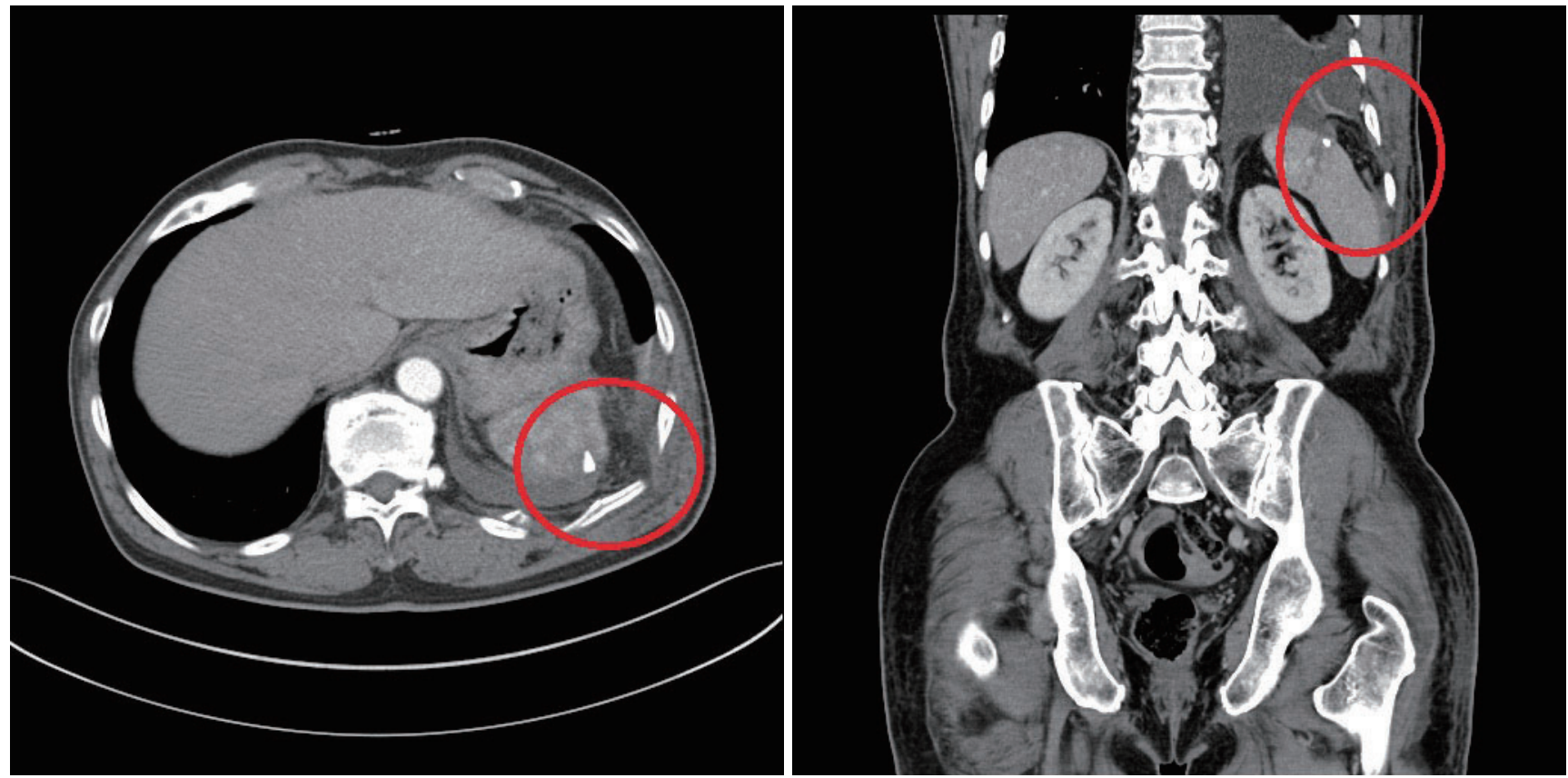

Fig. 2. Follow-up abdominopelvic computed tomography after 7 days, fat density increased in the thoracic cavity above spleen and retained a foreign body in the spleen (red circle).


Fig. 3. Intraoperative finding in video-assisted thoracic surgery, arrow indicate a foreign body.

ment is possible. However, delayed diagnosis of traumatic diaphragmatic rupture is associated with high morbidity and mortality in the long-term. It is recommended that thoracoscopy or laparoscopy is used for the diagnosis and repair of a missed diaphragmatic injury [2,3].

In cases with a retained foreign body in the peritoneal cavity, it can sometimes cause complications in the longterm, although it had not resulted in peritonitis or bleeding during the relatively short-term period. Some studies reported that a retained foreign body in a penetrating abdominal injury is a relative indication of laparotomy. In these selective patients, without other laparotomy indica- tions, a more efficient management policy can be chosen [4]. The retained foreign body in the peritoneal cavity which has not been removed in the acute phase might have resulted in unpredicted complication and a longterm follow-up should be required [5-7]. In this case, initial AP CT findings described the foreign body like a bony fragment and there was no peritonitis and other abdominal injuries, so conservative manage was decided.

The diaphragm is the border between the thoracic and the peritoneal cavity. In the acute phase of trauma, if diaphragmatic injury with concomitant injury of the thorax or the peritoneum is detected, it might be approached 
through the region of the concomitant injury, thoracotomy or laparotomy [8]. It is unclear what the better method of the approach of surgical treatment is applied to traumatic diaphragmatic injury in delayed diagnosis. In this case, it was planned to surgically repair the diaphragm injury through VATS because we noted retained hemothorax and suspected diaphragm injury without signs of peritonitis in the follow-up AP CT.

VATS has also shown accuracy in diagnosing and repairing diaphragm injuries if the intra-abdominal injury can be excluded along to clinical examination and radiologic investigations, in hemodynamically stable patients [9]. In our experience, the VATS approach is a reliable tool for the diagnosis and treatment of traumatic diaphragm injury, and although limited, can help explore the peritoneum through the lacerated site in the diaphragm.

This case, wherein a retained foreign body in the spleen with diaphragm rupture was successfully removed by VATS through the lacerated site of the diaphragm, shows the effectiveness of a minimally invasive approach to treat these injuries concomitantly with injury of the thorax, diaphragm, and peritoneal organ with a retained foreign body

\section{REFERENCES}

1. Mariadason JG, Parsa MH, Ayuyao A, Freeman HP. Management of stab wounds to the thoracoabdominal region. A clinical approach. Ann Surg 1988;207:335-40.

2. Mihos P, Potaris K, Gakidis J, Paraskevopoulos J, Varvatsoulis P, Gougoutas B, et al. Traumatic rupture of the diaphragm: experience with 65 patients. Injury 2003;34:169-72.

3. Sartipy U, Franneby U, Dellgren G, Hillebrant CG. Traumatic rupture of the diaphragm. Severe late complications of missed diaphragmatic rupture following a traffic accident 20 years ago. Lakartidningen 2004;101:288-9.

4. Clarke SC, Stearns AT, Payne C, McKay AJ. The impact of published recommendations on the management of penetrating abdominal injury. Br J Surg 2008;95:515-21.

5. Mendez LE, Medina C. Late complication of laparoscopic salpingoophorectomy: retained foreign body presenting as an acute abdomen. JSLS 1997;1:79-81.

6. Kalliakmanis V, Pikoulis E, Hitos A, Karavokyros IG, Gougoudi E, Leppaniemi A. A retained foreign body in the peritoneal cavity causing intestinal obstruction by intraluminal migration. Zentralbl Chir 2007;132:70-2.

7. Campbell JH, Efendy JL, Han C, Girjes AA, Campbell GR. Haemopoietic origin of myofibroblasts formed in the peritoneal cavity in response to a foreign body. J Vasc Res 2000;37:364-71.

8. Korymasov EA, Pushkin SI, Benian AS, Reshetov AP, Borkovskiĩ AI, Medvedchikov-Ardiia MA. Thoracoscopic suturing of diaphragm rupture and ribs osteosynthesis in patient with severe concomitant chest injury. Khirurgiia (Mosk) 2014:82-4.

9. Paci M, Ferrari G, Annessi V, de Franco S, Guasti G, Sgarbi G. The role of diagnostic VATS in penetrating thoracic injuries. World J Emerg Surg 2006;1:30. 